\title{
ON CYCLICITY IN WEIGHTED DIRICHLET SPACES
}

\section{F. JAFARI and R. RAPOSA}

(Received 6 August 1997 and in revised form 17 February 1998)

\begin{abstract}
We extend some results of Brown and Shields on cyclicity to weighted Dirichlet spaces $0<\alpha<1$. We prove a comparison theorem for cyclicity in these spaces and provide a result on the geometry of the family of cyclic vectors in general functional Hilbert spaces.
\end{abstract}

Keywords and phrases. Weighted Dirichlet spaces, cyclicity, functional Hilbert spaces.

1991 Mathematics Subject Classification. 30H05, 46E20, 47B37.

1. Introduction. In 1949, A. Beurling characterized the cyclic vectors for the operator of multiplication by $z$ on $H^{2}$. The important consequences of this work in function theory has led many investigators to study questions related to cyclicity on other Banach spaces of analytic functions.

In 1984, Brown and Shields [3] published an interesting paper giving extensive results and many open questions on cyclicity in Dirichlet space. Subsequently, Brown [2] and others [5, 6] have provided answers to some of these open questions and have extended these results to Bloch, Bergman and other spaces. Interesting applications of cyclicity to operator theory are given in [7].

Motivated by several of the open questions posed by Brown and Shields, in this paper, we consider the question of cyclicity on the weighted Dirichlet spaces. Our notation and terminology in this paper are standard. We use $\Delta$ to denote the open unit disk in the complex plane, $H^{2}$ to denote the Hardy space of order 2 , and $D_{\alpha}, 0<\alpha<1$, to denote

$$
\left\{f \in H^{2}: f(z)=\sum_{n=o}^{\infty} a_{n} z^{n}, \sum_{n=o}^{\infty}(n+1)^{\alpha}\left|a_{n}\right|^{2}<\infty\right\} .
$$

For $\alpha=1$, this is the classical Dirichlet space $D_{1}$ and, for $\alpha=0$, these space coincide with Hardy space $H^{2}$. We refer the reader to [3] for an extensive discussion of properties and a comparison of this interpolating family of Hilbert spaces with Bergman and other spaces. Significant differences between the property of these weighted spaces and the Dirichlet space are discussed in [1].

In Section 2 we present an example and some preliminary results which either motivate our development or they are needed later. In Section 3, we prove a basic estimate leading to Theorems 3.2 and 3.4. Theorem 3.2 asserts that if $f \in D_{\alpha}, 0<\alpha<1, g \in$ $H^{\infty} \cap D_{1}$, and $|f| \geq|g|$, then the cyclicity of $g^{2}$ in $D_{\alpha}$ implies that of $f$. Theorem 3.4 improves this result by replacing $g^{2}$ by $g$. An easy-to-check variation of Theorem 3.4 is given by Proposition 3.5. Finally, in Section 4, we provide a result on the structure of the family of cyclic vectors in functional Hilbert spaces. 
2. Preliminaries. A vector $x$ in a Hilbert space $H$ is a cyclic vector for the operator $T: H \rightarrow H$ if the orbit of $x$ under the action of polynomials in $T$ on $x$ is dense in $H$. It is well known that the cyclic vectors on $H^{2}$ for the operator of multiplication by $z$ are exactly the outer functions. Using the fact that $D_{\alpha} \rightarrow H^{2}$ is a continuous imbedding for $\alpha>0$, it is proved in [3] that a cyclic vector on $D_{\alpha}$ should be outer in $H^{2}$. Carleson [4] has constructed an outer function in $D_{1}$, which is not cyclic in $D_{1}$. By taking $E$ to be a closed set of capacity zero on the unit circle, Carleson constructs a function $f \in D_{1}$ such that the boundary values of $f$ vanish on $E$ but $f$ is not identically 0 . Clearly, this $f$ may be chosen to be outer. Carleson shows that the set of all functions $f \in D_{1}$ whose boundary values vanish on $E, D_{E}$, is a closed subspace of $D_{1}$. Hence, $[f] \subseteq D_{E} \subset D_{1}$, where $[f]$ denotes the norm closure of the polynomial multiples of $f$. Replacing the capacity by capacity of order $\alpha, C_{\alpha}, \alpha>0$, taking $E$ such that $C_{\alpha}(E)>0$, and $f$ as before, we obtain $[f] \subseteq\left(D_{\alpha}\right)_{E} \subset D_{\alpha}$. Thus, the cyclic vectors in $D_{\alpha}$ form a proper subset of the outer functions in $H^{2}$.

We also note that $f \in D_{\alpha}$ if and only if $f^{\prime} \in D_{\alpha-2}$ for $-\infty<\alpha<\infty$, and if $[f]=D_{\alpha}$, then $[f]=D_{\beta}$ for $\beta<\alpha$.

The proof of the following basic lemma appears in [3].

LEMMA 2.1. Let $f, g \in D_{\alpha},-\infty<\alpha<\infty$, and let $p$ be a polynomial. Then

(a) $p[f] \subset[f]$.

(b) If $g \in[f]$, then $[g] \subset[f]$.

(c) If $g \in[f]$ and $g$ is cyclic, then $f$ is cyclic.

(d) $f$ is cyclic if and only if there exist polynomials $\left\{p_{n}\right\}$ such that $p_{n} f \rightarrow 1$ (in norm).

Brown and Shields ask whether $f, g \in D_{\alpha}, g$ cyclic and $|f(z)| \geq|g(z)|$ for all $z \in \Delta$ implies that $f$ is cyclic.

They show that

Proposition 2.2. For $\alpha \in R \backslash(0,1]$, if $f, g \in D_{\alpha},|f(z)| \geq|g(z)|$ for all $z \in \Delta$ and $g$ is cyclic, then $f$ is cyclic.

With some additional hypotheses, [3, Thm. 1] also provides an affirmative answer to their question for $D_{1}$. We provide an analogous answer to their question for the weighted Dirichlet spaces $D_{\alpha}$ for $\alpha \in(0,1)$.

\section{Results}

LEMMA 3.1. Let $f \in D_{\alpha}, 0<\alpha<1$, and $g \in D_{1}$. Let $f_{t}(z)=f(t z), 0<t<1$. Then

$$
\int_{0}^{2 \pi} \int_{0}^{1}\left|f-f_{t}\right|^{2}\left|g_{t}^{\prime}\right|^{2}\left(1-r^{2}\right)^{-\alpha+1} r d r d \theta \leq C\|f\|_{\alpha}^{2}\|g\|_{1}^{2}
$$

Proof. Let $f(z)=\sum_{n=0}^{\infty} \hat{f}(n) z^{n}$. Then, if $|z|=r$, by the Cauchy-Schwarz inequality,

$$
\begin{aligned}
|f(z)-f(t z)|^{2} & \leq \sum_{n=0}^{\infty}(n+1)^{\alpha}|\hat{f}(n)|^{2} \sum_{n=0}^{\infty} \frac{r^{2 n}\left(1-t^{n}\right)^{2}}{(n+1)^{\alpha}} \\
& \leq\|f\|_{\alpha}^{2} \sum_{n=0}^{\infty} \frac{r^{n}\left(1-t^{n}\right)}{(n+1)^{\alpha}} \leq\|f\|_{\alpha}^{2} \sum_{n=0}^{\infty} \frac{r^{n}}{(n+1)^{\alpha}} \\
& \leq C\|f\|_{\alpha}^{2} \frac{1}{(1-r)^{1-\alpha}} .
\end{aligned}
$$


The last inequality follows directly from a formula by Euler for the gamma function (see [10, p. 237] for example). Hence, since $(1-r)^{1-\alpha}$ and $\left(1-r^{2}\right)^{1-\alpha}$ are comparable,

$$
\begin{gathered}
\int_{0}^{2 \pi} \int_{0}^{1}\left|f-f_{t}\right|^{2}\left|\left(g_{t}\right)^{\prime}\right|^{2}\left(1-r^{2}\right)^{1-\alpha} r d r d \theta \\
\leq C\|f\|_{\alpha}^{2} \int_{0}^{1}\left[\int_{0}^{2 \pi}\left|g^{\prime}(t z)\right|^{2} d \theta\right] r d r \\
=C\|f\|_{\alpha}^{2}\left\|g_{t}\right\|_{1}^{2} \leq C\|f\|_{\alpha}^{2}\|g\|_{1}^{2},
\end{gathered}
$$

where the metric in $D_{1}$ is, as usual, given by $\int_{0}^{2 \pi} \int_{0}^{1}\left|g^{\prime}\right|^{2} r d r d \theta$.

THEOREM 3.2. Suppose that $f \in D_{\alpha}, 0<\alpha<1, g \in H^{\infty} \cap D_{1}$, and $|f(z)| \geq|g(z)|$ for all $z \in \Delta$. If $g^{2}$ is cyclic in $D_{\alpha}$, then $f$ is cyclic in $D_{\alpha}$.

Proof. Let $g_{t}=g(t z)$ for $0<t<1$. Since $\left(g_{t}^{2} / f_{t}\right)$ is a multiplier of $D_{\alpha},\left(g_{t}^{2} / f_{t}\right) f \in$ [f]. We show that these functions converge in norm to $g^{2}$ as $t \rightarrow 1$. Hence, $g^{2} \in[f]$. Clearly, this implies that $[f] \supseteq\left[g^{2}\right]=D_{\alpha}$ by hypothesis. We start by noting that

$$
\left\|\frac{g_{t}^{2}}{f_{t}} f-g^{2}\right\|_{\alpha} \leq\left\|\frac{g_{t}^{2}}{f_{t}} f-g_{t}^{2}\right\|_{\alpha}+\left\|g_{t}^{2}-g^{2}\right\|_{\alpha} .
$$

Clearly, the second term tends to 0 as $t \rightarrow 1$. So, it suffices to prove that $\left(g_{t}^{2} / f_{t}\right) f \rightarrow g_{t}^{2}$ in $D_{\alpha}$-norm. This is equivalent to showing that the derivative of $\left(g_{t}^{2} / f_{t}\right) f-g_{t}^{2} \rightarrow 0$ in $D_{\alpha-2}$ as $t \rightarrow 1$. Write

$$
\left(\frac{g_{t}^{2}}{f_{t}} f-g_{t}^{2}\right)^{\prime}=\left(g_{t}^{2}\left(\frac{f-f_{t}}{f_{t}}\right)\right)^{\prime}=g_{t}^{2}\left(\frac{f-f_{t}}{f_{t}}\right)^{\prime}+2 g_{t}\left(g_{t}\right)^{\prime}\left(\frac{f-f_{t}}{f_{t}}\right)=\phi_{1}+\phi_{2} \text {. }
$$

By hypothesis,

$$
\left|\frac{\phi_{2}}{2}\right| \leq\left|\frac{g_{t}}{f_{t}}\right|\left|g_{t}^{\prime}\right|\left|f-f_{t}\right| \leq\left|g_{t}^{\prime}\right|\left|f-f_{t}\right|
$$

Hence,

$$
\left\|\frac{\phi_{2}}{2}\right\|_{\alpha-2} \leq \int_{0}^{2 \pi} \int_{0}^{1}\left|g_{t}^{\prime}\right|^{2}\left|f-f_{t}\right|^{2}\left(1-r^{2}\right)^{1-\alpha} r d r d \theta
$$

To show that (3.7) tends to zero as $t \rightarrow 1$, note that if $f$ is a polynomial (in fact, if $f$ is in the disk algebra) and $g$ is arbitrary, then the convergence to zero is clear. For arbitrary $f$, given $\varepsilon>0$, choose a polynomial $p$ such that $\|f-p\|_{\alpha}<\varepsilon$ and let $h=f-p$. Then

$$
\begin{aligned}
\int_{0}^{2 \pi} \int_{0}^{1} \mid f & -\left.f_{t}\right|^{2}\left|\left(g_{t}\right)^{\prime}\right|^{2}\left(1-r^{2}\right)^{1-\alpha} r d r d \theta \\
& =\int_{0}^{2 \pi} \int_{0}^{1}\left|h+p-h_{t}-p_{t}\right|^{2}\left|\left(g_{t}\right)^{\prime}\right|^{2}\left(1-r^{2}\right)^{1-\alpha} r d r d \theta \\
\leq & 2 \int_{0}^{2 \pi} \int_{0}^{1}\left|h-h_{t}\right|^{2}\left|\left(g_{t}\right)^{\prime}\right|^{2}\left(1-r^{2}\right)^{1-\alpha} r d r d \theta \\
& +2 \int_{0}^{2 \pi} \int_{0}^{1}\left|p-p_{t}\right|^{2}\left|\left(g_{t}\right)^{\prime}\right|^{2}\left(1-r^{2}\right)^{1-\alpha} r d r d \theta
\end{aligned}
$$


By the above remarks, the second term tends to zero as $t \rightarrow 1$. On the other hand, by Lemma 3.1, we have

$$
\lim _{t \rightarrow 1} \int_{0}^{2 \pi} \int_{0}^{1}\left|f-f_{t}\right|^{2}\left|\left(g_{t}\right)^{\prime}\right|^{2}\left(1-r^{2}\right)^{1-\alpha} r d r d \theta \leq C\|h\|_{\alpha}^{2}\|g\|_{1}^{2} \leq C \varepsilon^{2}\|g\|_{1}^{2} .
$$

Substituting this result in (3.7), we obtain $\left\|\phi_{2}\right\|_{\alpha-2}<C \varepsilon^{2}\|g\|_{1}^{2}$.

Next, to show that $\left\|\phi_{1}\right\|_{\alpha-2} \rightarrow 0$, we write

$$
\phi_{1}=g_{t}^{2}\left(\frac{f-f_{t}}{f_{t}}\right)^{\prime}=\frac{g_{t}^{2} f_{t}}{f_{t}^{2}}\left(f-f_{t}\right)^{\prime}-\frac{g_{t}^{2}}{f_{t}^{2}} f_{t}^{\prime}\left(f-f_{t}\right)=\phi_{3}+\phi_{4}
$$

Clearly, $\left|\phi_{4}\right| \leq\left|\left(f-f_{t}\right)\right|\left|f_{t}^{\prime}\right|$. So, $\left\|\phi_{4}\right\|_{\alpha-2} \rightarrow 0$ as $t \rightarrow 1$.

Likewise, $\left|\phi_{3}\right| \leq\left|\left(f-f_{t}\right)^{\prime} g_{t}\right|$. Thus, $\left\|\phi_{3}\right\|_{\alpha-2} \leq\left\|\left(f-f_{t}\right)^{\prime}\right\|_{\alpha-2}\left\|g_{t}\right\|_{\infty}$ and $\left\|\phi_{3}\right\|_{\alpha-2} \rightarrow$ 0 as $t \rightarrow 1$. This completes the proof.

The following corollary follows immediately from Theorem 3.2.

COROLlarY 3.3. If $f \in D_{\alpha}, 0<\alpha<1$, and $|f(z)| \geq \delta>0$ for all $z \in \Delta$, then $f$ is cyclic.

Proof. Simply let $g(z)=\delta$ in Theorem 3.2.

Brown and Shields also asked [3, Question 8], whether $g$ needs to be only cyclic and not $g^{2}$. The following two results provide an affirmative answer in the context of our development.

THEOREM 3.4. If $g \in D_{1} \cap H^{\infty}, g$ is cyclic in $D_{\alpha}$ for $0<\alpha<1, f \in D_{\alpha}$, and $|f(z)| \geq$ $|g(z)|$ for all $z \in \Delta$, then $f$ is cyclic.

Proof. By [8, Thm. 1.7(a)] (or [9, Thm. 3.2]), $D_{1} \cap H^{\infty}$ is contained in the set of multipliers of $D_{\alpha}$. Hence, $g^{2} \in D_{\alpha} \cap H^{\infty}$. Since $g$ is cyclic in $D_{\alpha}$, by [3, Prop. 8], $g^{2}$ is cyclic in $D_{\alpha}$. Now, using Theorem 3.2, it follows that $f$ is cyclic in $D_{\alpha}$.

Finally, the following proposition provides a variant of the above under the readily tested hypothesis $g^{\prime} \in H^{\infty}$.

Proposition 3.5. If $g$ is cyclic in $D_{\alpha}$ and $g^{\prime} \in H^{\infty}$, then $g^{2}$ is cyclic in $D_{\alpha}$ for $0<$ $\alpha<1$.

Although we are able to provide a proof similar to those of Theorems 3.2 and 3.4, we demonstrate an alternative proof.

Proof. Plainly, if $g^{\prime} \in H^{\infty}$, then $g \in D_{\alpha} \cap H^{\infty}$ for all $\alpha>0$. Also, $g^{2} \in H^{\infty}$ and if $d A_{\alpha}=\left(1-r^{2}\right)^{-1+\alpha} r d r d \theta$, then

$$
\int_{\Delta}\left|\left(g^{2}\right)^{\prime}\right|^{2} d A_{\alpha}=4 \int_{\Delta}|g|^{2}\left|g^{\prime}\right|^{2} d A_{\alpha} \leq 4\|g\|_{\infty}^{2} \int_{\Delta}\left|g^{\prime}\right|^{2} d A_{\alpha}=4\|g\|_{\infty}^{2}\|g\|_{\alpha}^{2}<\infty .
$$

So, $g^{2} \in D_{\alpha} \cap H^{\infty}$. By hypothesis, since $g$ is cyclic in $D_{\alpha}$, by Lemma 2.1 , there exist polynomials $\left\{p_{n}\right\}$ such that $p_{n}(z) g(z) \rightarrow 1$ for all $z \in \Delta$ and $\left\|p_{n} g\right\|_{\alpha} \leq C$. To show that $g^{2}$ is cyclic, we show that $p_{n}(z) g^{2}(z) \rightarrow g(z)$ pointwise and $\left\|p_{n} g^{2}\right\|_{\alpha} \leq C$. 
Fix $z \in \Delta$. Since $p_{n}(z) g(z) \rightarrow 1$ pointwise, given $\varepsilon>0$, there exists $N$ such that

$$
\left|p_{n}(z) g(z)-1\right|<\frac{\varepsilon}{\|g\|_{\infty}} \quad \forall n>N .
$$

Then for $n>N$

$$
\left|p_{n}(z) g^{2}(z)-g(z)\right| \leq\|g\|_{\infty}\left|p_{n}(z) g(z)-1\right|<\frac{\varepsilon}{\|g\|_{\infty}}=\varepsilon .
$$

So, $p_{n}(z) g^{2}(z) \rightarrow g(z)$ pointwise. Now, Consider $p_{n} g^{2}=\left(p_{n} g\right) g$. Then,

$$
\begin{aligned}
\left\|p_{n} g^{2}\right\|_{\alpha}^{2} & \leq 2\left(\int_{\Delta}\left|\left(p_{n} g\right)^{\prime} g\right|^{2} d A_{\alpha}+\int_{\Delta}\left|\left(p_{n} g\right) g^{\prime}\right|^{2} d A_{\alpha}\right) \\
& \leq 2\|g\|_{\infty}^{2}\left\|p_{n} g\right\|_{\alpha}^{2}+2 \int_{\Delta}\left|\left(p_{n} g\right) g^{\prime}\right|^{2} d A_{\alpha} .
\end{aligned}
$$

Since $g^{\prime} \in H^{\infty}$, the result follows.

4. The geometry of cyclic vectors. In this section, we analyze the structure of the set of cyclic vectors $C$ for an operator $T$ on a functional Hilbert space $H$. As usual, a functional Hilbert space refers to a separable Hilbert space of analytic functions in which the polynomials are dense. Clearly, the results of this section apply to the weighted Dirichlet spaces of analytic functions. The following results provide the core argument.

Proposition 4.1. Let $H$ be a functional Hilbert space and $C$ be the set of cyclic vectors for $T$ in $H$. Then $H=C+C$.

Proof. First, we show that $C$ is a dense $G_{\delta}$ set in $H$. Let $U_{n}$ be a countable base for the topology of $H$ and $Q_{n}(T)=\cup_{p \in P}[p(T)]^{-1}\left(U_{n}\right)$, where $P$ is the set of polynomials. Clearly, $Q_{n}$ is open and nonempty. We show that $\cap_{n} Q_{n}$ is precisely the set of cyclic vectors for $T$. If $x \in \cap_{n=1}^{\infty} Q_{n}$, then $x \in Q_{n}$ for all $n$. Hence, there exist polynomials $p_{n}(z)$ such that $p_{n}(T) x \in U_{n} \forall n$. Therefore, $x$ is a cyclic vector for $T$. Also, if $x$ is cyclic for $T$, then, clearly, $x \in \cap_{n=1}^{\infty} Q_{n}$. Hence, the set of cyclic vectors for $T$ is a $G_{\delta}$ set.

To show that $\cap_{n} Q_{n}$ is nonempty, we show that each $Q_{n}$ is dense. Then, by the Baire Category theorem, $\cap_{n} Q_{n}$ is a dense $G_{\delta}$ set. Let $h \in H$ and $\varepsilon>0$. We want to show that there exist $y \in Q_{n}$ such that $\|y-h\|<\varepsilon$. Let $U_{n}=B_{H}(0, \delta)$, the ball of radius $\delta$ in $H$ centered at the origin. Let $M=\|T\|$ and $p(z)=(\delta / 2 M) z$. Then $p(T)$ maps the unit disk into $B_{H}(0, \delta)$ for if $z \in \Delta$, then

$$
\|p(T)(z)\|=\left\|\frac{\delta}{2 M} T\right\|<\delta .
$$

Now, let $x \in H$ and consider $(C+x) \cap C$. This set is a dense $G_{\delta}$ set in $H$ and, thus, $(C+x) \cap C \neq \emptyset$. Hence, there exist $y \in H$ such that $y \in(C+x) \cap C$. So, $y$ is cyclic and $y=h+x$ for some $h \in C$. Then $x=y+(-h) \in C+C$.

It is interesting to ask if the set of cyclic vectors in $H$ is stable under small perturbations. For spaces contained in the Hardy space, this is, of course, more meaningful if we restrict to the class of outer functions. The following result presents a negative answer. Let $C_{S}=C \cap\left\{f \in C:\|f\|_{H}=1\right\}$ and $A=\{f \in H: 1-\varepsilon<\|f\|<1+\varepsilon\}$. 
THEOREM 4.2. Fix $\varepsilon>0$ and let $A=\{f \in H: 1-\varepsilon<\|f\|<1+\varepsilon\}$. Then $C_{S}+\varepsilon C_{S}=A$.

Proof. Clearly, $C_{S}$ is a dense $G_{\delta}$ subset of the unit sphere of $H$. Hence, by essentially the same argument as in Proposition 4.1, the assertion follows.

COROLlary 4.3. The class of cyclic vectors in $D_{\alpha}$ is unstable. That is, given $x \in$ $C \cap D_{\alpha}$ and $\varepsilon>0$, there exists $y \in D_{\alpha},\|y\|<\varepsilon$ such that $x+y$ is not a cyclic vector.

ACKNOWLEDGEMENT. The authors wish to thank Professor Leon Brown for helpful discussions and the referee for pointing out several improvements.

\section{REFERENCES}

[1] D. Betebenner, L. Brown, and F. Jafari, On the range of functions belonging to weighted Dirichlet spaces, submitted.

[2] L. Brown, Invertible elements in the Dirichlet space, Canad. Math. Bull. 33 (1990), no. 4, 419-422. MR 92e:46054. Zbl 741.30040.

[3] L. Brown and A. L. Shields, Cyclic vectors in the Dirichlet space, Trans. Amer. Math. Soc. 285 (1984), no. 1, 269-303. MR 86d:30079. Zbl 537.30040.

[4] L. Carleson, Sets of uniqueness for functions regular in the unit circle, Acta Math. 87 (1952), 325-345. MR 14,261a. Zbl 046.30005.

[5] B. Korenblum, Outer functions and cyclic elements in Bergman spaces, J. Funct. Anal. 115 (1993), no. 1, 104-118. MR 94h:46038. Zbl 780.30028.

[6] S. Richter, Invariant subspaces of the Dirichlet shift, J. Reine Angew. Math. 386 (1988), 205-220. MR 89e:47048. Zbl 635.46021.

[7] A. L. Shields, Weighted shift operators and analytic function theory, Topics in operator theory (Providence, RI), Math. Surveys, no. 13, Amer. Math. Soc., 1974, pp. 49-128. MR 50 14341. Zbl 303.47021.

[8] D. A. Stegenga, Multipliers of the Dirichlet space, Illinois J. Math. 24 (1980), no. 1, 113-139. MR 81a:30027. Zbl 432.30016.

[9] R. S. Strichartz, Multipliers on fractional Sobolev spaces, J. Math. Mech. 16 (1967), 10311060. MR 35\#5927. Zbl 145.38301.

[10] E. T. Whitaker and G. N. Watson, A Course in Modern Analysis, 3rd ed., Cambridge University Press, London, 1920.

JAFARI: DEPARTMENT OF MATHEMATICS, UNIVERSITY OF WYOMING, LARAMIE, WY 82071-3036, USA

Raposa: Department of Math and CS, Montana State University, Billings, MT 59101, USA 


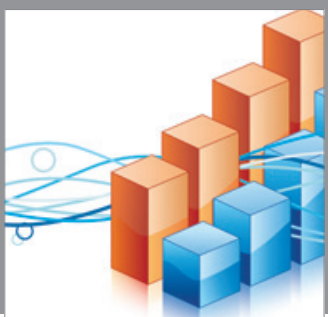

Advances in

Operations Research

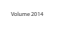

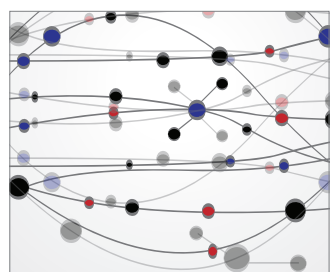

\section{The Scientific} World Journal
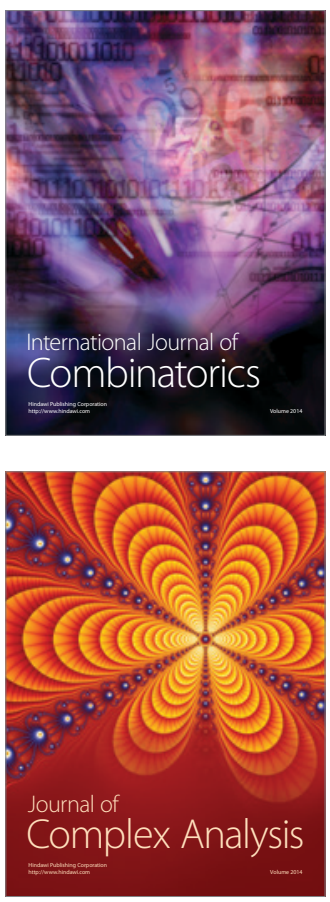

International Journal of

Mathematics and

Mathematical

Sciences
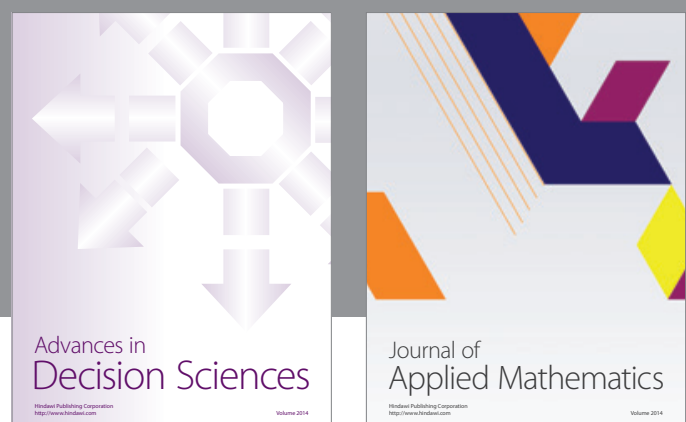

Journal of

Applied Mathematics
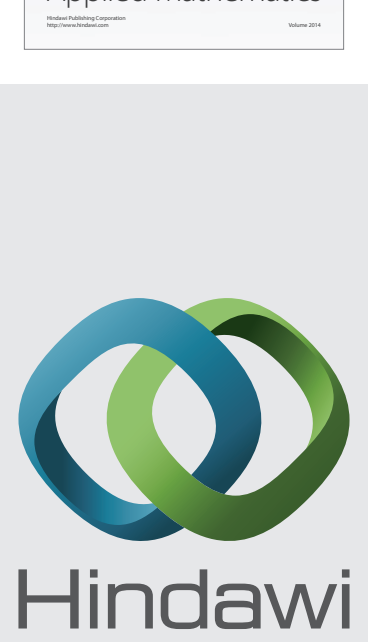

Submit your manuscripts at http://www.hindawi.com
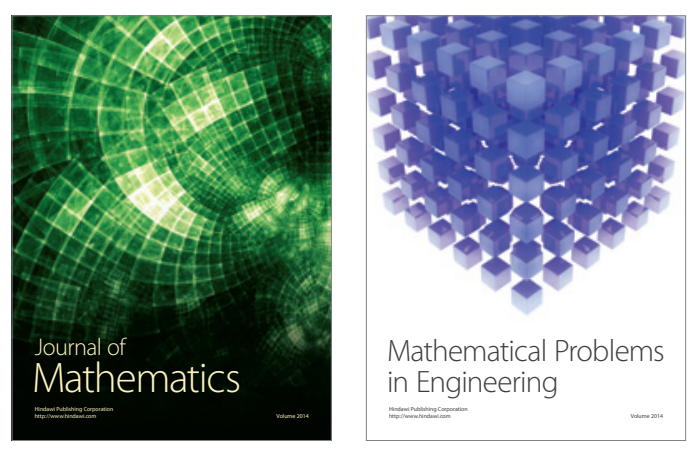

Mathematical Problems in Engineering
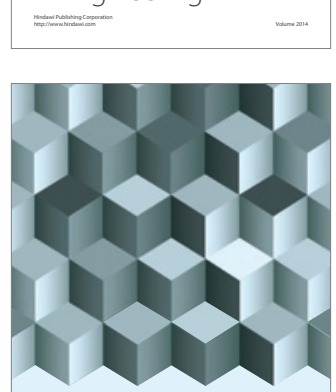

Journal of

Function Spaces
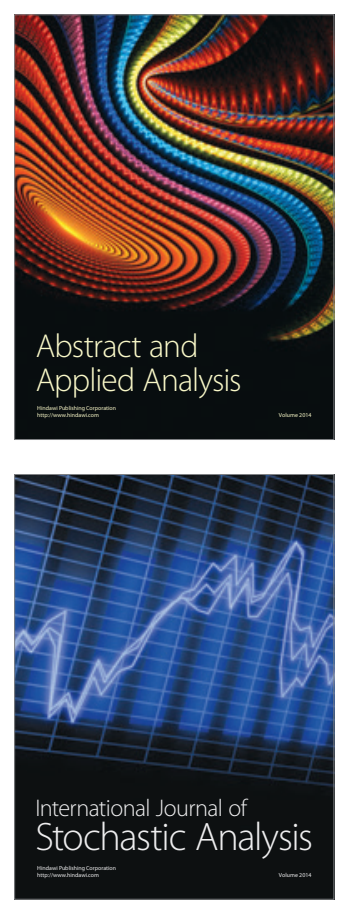

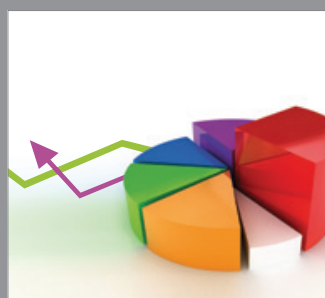

ournal of

Probability and Statistics

Promensencen
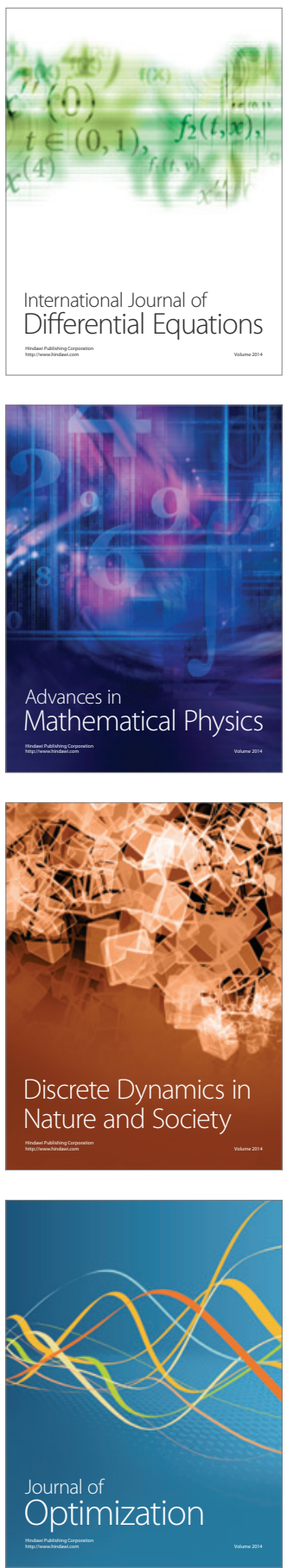